\title{
Biotherapeutics and immunogenicity: ophthalmic perspective
}

\author{
Ashish Sharma ${ }^{1} \cdot$ Nilesh Kumar $^{1} \cdot$ Baruch D. Kuppermann ${ }^{2} \cdot$ Francesco Bandello $^{3} \cdot$ Anat Loewenstein $^{4}$
}

Received: 14 March 2019 / Accepted: 28 March 2019 / Published online: 9 April 2019

(c) The Royal College of Ophthalmologists 2019

Biotherapeutics have revolutionized the treatment of many retinal pathologies since their inception by their virtue of directly disrupting the pathophysiological mechanism of the diseases, such as age-related macular degeneration (AMD), diabetic macular edema (DME), retinal venous occlusion (RVO), etc. [1]. The attributable blindness to AMD has been reduced by $50-72 \%$ since the commercial introduction of anti-vascular endothelial growth factor (VEGF) agents for its treatment $[2,3]$. The innovator anti-VEGF molecules such as Bevacizumab, Ranibizumab, and Aflibercept, and their biosimilar (Razumab (Ranibizumab), India) are the current therapeutic options available in the market and are having high level of efficacy for the indications they are approved for. There are though reports of these proteins causing immunogenic reactions upon intra-vitreal injection [4-6].) These reactions are being attributed to the batch to batch variability in production and storage of biologics [7]. But it is also pertinent to understand that these reactions are a complex interplay between various drug and host factors. This immunogenicity raises question that how this immune reaction to a drug is taking place in an immune privileged organ like eye where adaptive immune reaction is not in play under normal circumstances.

The initial reactions to systemic administration of biotherapeutic drugs were attributed to the adjuvants of the injections [8]. It was then disproved by eliciting immune

$\triangle$ Ashish Sharma

drashish79@hotmail.com

1 Department of Vitreoretina, Lotus Eye Hospital and Institute, Coimbatore, Tamil Nadu, India

2 Gavin Herbert Eye Institute, University of California, Irvine, Irvine, CA, USA

3 Department of Ophthalmology, University Vita-Salute, Scientific Institute San Raffaele, Milano, Italy

4 Division of Ophthalmology, Tel Aviv Sourasky Medical Center and Sackler Faculty of Medicine, Tel Aviv University, Tel Aviv, Israel reaction upon injection of pure proteins and was attributed to the chimeric non-self-nature of the molecules. This hypothesis again failed when fully humanized therapeutic proteins were also reported to elicit reactions, though at a lower rate [9]. These immune responses have led to discovery of anti-drug antibodies (ADA), which are postulated to be developing against the freely available monoclonal antibodies due to their inherent protein structure. They are produced in two variants, epitope neutralizing ADA (antiidiotypic ADA) and non-neutralizing ADA (binding ADA) against the backbone [10]. These neutralizing ADA compete and bind to the antigen-binding site and thus bring down amount of freely available drug, producing treatment failure or resistance. The more severe effect these ADA are postulated to induce is hypersensitivity or anaphylactic reactions to biotherapeutics, which cause devastating phenomenon like sterile endophthalmitis upon intra-vitreal injections.

It is very important to understand that these ADA are induced by the complex nature of biotherapeutics and thus more complex molecules will theoretically have a higher incidence of adverse drug reactions (ADR). The almost double incidence of endophthalmitis in patients taking Aflibercept injections when compared to Bevacizumab or Ranibizumab is a witness to this hypothesis [4, 5]. Future research may be required to find out methods to decrease ADR with rising complexity of molecules.

These ADA have been recruited in the in vitro analysis of immunogenicity of the drugs during their development by various commercially available assays [11]. In the era where biosimilars are on the front runners in development of new agents, it is pertinent to establish the similar immunogenicity safety of these agents [12]. This basic criterion is one of the most difficult parameters to be evaluated because of the nature of assays and limited availability of ADA. While the regulators have included establishment of comparative immunogenicity in the guidelines, there is no quantification of the allowed deviation in the parameters. No two assays are similar in nature and their minimum cut-off parameters differ widely [13]. 
The ADA are usually found in bound state with these drugs in human serum and so free ADA is available in a very limited quantity, and thus large trials establishing similar efficacy on different assays is not possible. While there are advances in these assays to evaluate presence of bound ADA even in low concentrations, they are not standardized, validated, or globally available yet. The regulatory authorities should mandate a head-to-head establishment of equivalency for each biosimilar with its reference molecule using same assay.

The immune privilege of eye was established with anatomical demonstration of blood ocular barriers and activation of retinal innate immunity in response to foreign antigens. Demonstration of ADA in patient's serum who took intra-vitreal anti-VEGF injections was attributed to be in response to the molecules which entered systemic circulation via aqueous drainage pathways. Intra-vitreal presence of these ADA have now been established and confirms presence of adaptive immunity in the eye in certain pathologies like AMD [14].

AMD is now being evaluated as an auto-immune condition where production of VEGF is a consequence of derailed innate and adaptive immunity $[15,16]$.The breach of internal limiting membrane (ILM) in neovascular AMD, with recruitment of inflammatory cells at the site of breach, thus exposes the vitreous cavity to hypersensitive immune system. Heritability of AMD is now gaining evidence based on its auto-immune pathogenesis [16]. With production ADA also linked to the polymorphism in human leukocyte antigens (HLA), it is logical to anticipate a genetic predisposition to treatment resistance and hypersensitive reactions to anti-VEGF [17]. As a clinician, this becomes very important to understand and implement in day to day practice. With variety of biologics and biosimilars available at disposal, the choice of therapy should strictly be for the proven indications. Unjustified injections might induce ADA and lead to treatment failure when it actually will be required. Also, use of molecules who share similar structure cannot be justified, if one of them has been proven to be ineffective, or has a secondary treatment failure, and might put the patient on risk of a hypersensitive reaction.

\section{What the future beholds?}

It is prudent that immunogenic safety of all the biotherapeutics and especially biosimilars are well validated. The regulators should implement post-marketing surveillance measures to ensure that no ADR should happen due to the adjuvants or contamination. With evaluation of reasons of treatment failure, it is now being established that the diseases being treated with biotherapeutics are multifactorial. The role of immunity in failure and ADR is also gaining evidence. With advent of more complex biologics, these reactions may render the drug unsafe. This has led to innovators evaluate a fixed drug combination, where an intra-vitreal immuno-modulator is injected along with biotherapeutic molecule which will supress the induction of ADA, as well as help in treating the immunological part of the disease itself. Also, it will be clinically unviable to evaluate each patient for genetic predisposition or send for ADA assays. The intra-dermal test dose is being evaluated with various dilutions for biologics in systemic use [18]. Future will unfold whether adoption of such tests can be of some role prior to intra-vitreal biologics.

\section{Compliance with ethical standards}

Conflict of interest The authors declare that they have no conflict of interest.

Publisher's note: Springer Nature remains neutral with regard to jurisdictional claims in published maps and institutional affiliations.

\section{References}

1. Yorston D. Anti-VEGF drugs in the prevention of blindness. Community Eye Health. 2014;27:44-6.

2. Bloch SB, Larsen M, Munch IC. Incidence of legal blindness from age-related macular degeneration in Denmark: year 2000 to 2010. Am J Ophthalmol. 2012;153:209-13.e2.

3. Bressler NM, Doan QV, Varma R, Lee PP, Suñer IJ, Dolan C, et al. Estimated cases of legal blindness and visual impairment avoided using ranibizumab for choroidal neovascularization: nonhispanic white population in the United States with age-related macular degeneration. Arch Ophthalmol [Internet]. 2011;129. http://archopht.jamanetwork.com/article.aspx? https://doi.org/10. 1001/archophthalmol.2011.140. Accessed 26 Feb 2019.

4. Kiss S, Dugel PU, Khanani AM, Broder MS, Chang E, Sun GH, et al. Endophthalmitis rates among patients receiving intravitreal anti-VEGF injections: a USA claims analysis. Clin Ophthalmol. 2018;12:1625-35.

5. Souied EH, Dugel PU, Ferreira A, Hashmonay R, Lu J, Kelly SP. Severe ocular inflammation following ranibizumab or aflibercept injections for age-related macular degeneration: a retrospective claims database analysis. Ophthalmic Epidemiol. 2016;23:71-9.

6. EXCLUSIVE: Complaints prompt Intas to recall batch of biosimilar Razumab in India-Moneycontrol.com [Internet]. https://www.moneycontrol.com/news/business/companies/ exclusive-complaints-prompt-intas-to-recall-batch-of-biosimilarrazumab-in-india-2251273.html. Accessed 8 Mar 2019.

7. Agrawal S, Joshi M, Christoforidis JB. Vitreous inflammation associated with intravitreal anti-VEGF pharmacotherapy. Mediators Inflamm. 2013;2013:1-6.

8. Janeway CA. Approaching the asymptote? Evolution and revolution in immunology. Cold Spring Harb Symp Quant Biol. 1989;54:1-13.

9. Hwang WYK, Foote J. Immunogenicity of engineered antibodies. Methods. 2005;36:3-10.

10. Garcês S, Demengeot J. The immunogenicity of biologic therapies. In: Puig L, Gulliver W, editors. Current problems in dermatology [Internet]. S. Karger AG; 2018. p. 37-48. https://www. karger.com/Article/FullText/478077. Accessed 26 Feb 2019. 
11. Schreitmüller T, Barton B, Zharkov A, Bakalos G. Comparative immunogenicity assessment of biosimilars. Future Oncol. 2019; 15:319-29.

12. Pineda C, Castañeda Hernández G, Jacobs IA, Alvarez DF, Carini C. Assessing the immunogenicity of biopharmaceuticals. BioDrugs Clin Immunother Biopharm Gene Ther. 2016;30:195-206.

13. Reinivuori $\mathrm{T}$, Kurki $\mathrm{P}$, Chamberlain P. Immunogenicity assessment of biosimilars. Pharm Med. 2018;32:103-21.

14. Wessels U, Zadak M, Reiser A, Brockhaus J, Ritter M, Abdolzade-Bavil A, et al. Immunogenicity testing of therapeutic antibodies in ocular fluids after intravitreal injection. Bioanalysis. 2018;10:803-14.
15. Camelo S. Potential sources and roles of adaptive immunity in age-related macular degeneration: shall we rename AMD into autoimmune macular disease? Autoimmune Dis. 2014; 2014:1-11.

16. Ambati J, Atkinson JP, Gelfand BD. Immunology of age-related macular degeneration. Nat Rev Immunol. 2013;13:438-51.

17. Vultaggio A, Petroni G, Pratesi S, Nencini F, Cammelli D, Ferraro A, et al. How the immune system responds to therapeutic biological agents. J Int Med Res. $2016 ; 44(1$ Suppl):38-42.

18. Khan DA. Hypersensitivity and immunologic reactions to biologics: opportunities for the allergist. Ann Allergy Asthma Immunol. 2016;117:115-20. 